valve involvement $(5.10 \%)$ and epididymitis (9.95\%). Among female patients, the frequency of genital ulcers is significantly higher than that of men $(86.24 \%$ vs. $68.69 \%$, $\mathrm{P}<0.001$ ).

Conclusion: We described the clinical characteristics of a large cohort of pediatric BD patients in China. While the clinical manifestations are largely similar to adult $\mathrm{BD}$, the time from symptom onset to diagnosis is significantly delayed in pediatric patients. Our study highlights the need to improve awareness of BD among pediatric providers to facilitate rapid diagnosis and treatment in children. REFERENCES:

[1] Yıldız et al. Pediatric Behçet's disease- clinical aspects and current concepts. Eur J Rheumatol 2020; 7(Suppl 1): S38-S47.

[2] Koné-Paut. Behçet's disease in children, an overview. Pediatric Rheumatology (2016) 14:10.

Table 1. Comparison of clinical features of BD in children and adults

\begin{tabular}{lccc}
\hline & $\begin{array}{c}\text { Children } \\
(\mathrm{n}=848)\end{array}$ & $\begin{array}{c}\text { Adult } \\
(\mathrm{n}=1531)\end{array}$ & $\mathrm{P}$ \\
\hline Age of first symptom $(\mathrm{y})$ & $11.59 \pm 4.11$ & $27.08 \pm 7.91$ & \\
Sex(M/F) & $412 / 436$ & $6.73 \pm 33.21$ & $<0.001$ \\
Years of diagnosis $(\mathrm{y})$ & $13.34 \pm 9.13$ & $1518(99.15 \%)$ & 0.007 \\
Recurrent oral aphthosis & $848(100 \%)$ & $1152(75.24 \%) 379$ & 0.176 \\
Genital Ulcers & $659(77.71 \%) 189$ & $508(33.18 \%) 1023$ & 0.134 \\
Ocular signs & $256(30.19 \%) 592$ & $1020(66.62 \%) 511$ & 0.132 \\
Cutaneous signs & $539(63.56 \%) 309$ & $82(5.36 \%) 1449$ & 0.421 \\
Neurological signs & $39(4.60 \%) 809$ & $244(15.94 \%) 1387$ & 0.001 \\
Gastrointestinal signs & $170(20.05 \%) 678$ & $162(10.58 \%) 1369$ & 0.542 \\
Vascular signs & $83(9.79 \%) 765$ & $110(7.18 \%) 1421$ & $<0.001$ \\
Heart valve signs & $31(3.66 \%) 817$ & $22(1.44 \%) 1509$ & $<0.001$ \\
Joint signs & $275(32.43 \%) 573$ & $347(22.66 \%) 1184$ & 0.013 \\
Fever & $3(0.35 \%) 845$ & & 0.381 \\
Positive pathergy test & $179(21.11 \%) 669$ & & \\
\hline
\end{tabular}

Acknowledgements: China Behcet' Disease Patients Assossiation.

Disclosure of Interests: None declared.

DOI: 10.1136/annrheumdis-2021-eular.3621

\begin{tabular}{|l|l}
\hline POS0082 & PERFORMANCES OF THE RISK FACTORS AND \\
PEDIATRIC VASCULITIS ACTIVITY SCORES \\
FOR DISEASE SEVERITY IN CHILDREN WITH \\
MULTISYSTEM INFLAMMATORY SYNDROME
\end{tabular}

B. Sözeri ${ }^{1}$, Ş. Çağlayan ${ }^{1}$, A. Akyol ${ }^{2}$, K. Ulu ${ }^{1}$, T. Coşkuner $^{1}$, C. Hasbal Akkuş ${ }^{2}$, F. Demir ${ }^{1} .{ }^{1}$ Health Sciences University, Umraniye Traning and Research Hospita, Pediatric rheumatology, Istanbul, Turkey; ${ }^{2}$ Health Sciences University, Umraniye Traning and Research Hospita, Pediatrics, Istanbul, Turkey

Background: Multisystem Inflammatory Syndrome in Children (MIS-C) is observed by hyperinflammation and cytokine storm. The spectrum of severity ranged from standard hospitalization to pediatric intensive care unit management. There is no specific activity score that predicts whether this hyperinflammatory state will be severe or result in mortality in pediatric patients. There are activity scores used in KD and other vasculitis such as Kobayashi score (KS) and Pediatric Vasculitis Activity Score (PVAS) that determine the severity of the disease in children.

Objectives: Based on the clinical similarity of MIS-C to these disease groups, we wanted to evaluate the performance of these disease activity scores. Also, we aimed to identify the factors associated with the disease severity of patients with MISC

Methods: We retrospectively enrolled a single-center cohort of 45 consecutive pediatric patients with MISC admitted to Umraniye Training and Resrach Hospital, Pediatric Rheumatology Clinic, Istanbul, Turkey, from April 20 to December 31, 2020. Medical information of each patient including demographic data, clinical characteristics, laboratory results, and outcomes was extracted retrospectively through review of electronic medical records. We analyzed all score systems including KS, PVAS, NLR, cHIS, and C-reactive protein/albumin ratio (CAR) as assessment factors for diagnosis for severe disease and evaluation of disease activity in MISC. All scores were compared between two groups and receiver operating characteristic (ROC) curve analysis was performed to evaluate diagnostic utility.

Results: We reported 45 patients (10 female, 35male) with MISC. Their mean age was $9.65 \pm 4.93$ years ( 7 months -18 years). All patients had fever (median 4 days), $71 \%$ patients had acute gastrointestinal symptoms, $37.8 \%$ of patient's conjunctivitis and only 5 patients had respiratory findings at admission. Twenty-four (46.7\%) patients met criteria for classic KD. Macrophage activation syndrome and myocardial dysfunction with or without cardiogenic shock were seen 14 and 10 patients respectively. All the patients had positive serology for SARSCoV-2, abnormal complete blood counts and coagulation tests, and elevated inflammatory markers.

We divided the disease severity into a moderate or severe group based on admission on intensive care unit (ICU). There were 15 patients with severe illness
(33\%). The median age of these patients was significantly older (11.3 years vs 9.16 years, $p=0.05)$. The median hospital stay period was 10 days. The median need for intensive care was on the first day (1-14th days), and the median lasted 5 (1-9) days. The majority of MISC patients were on Intravenous immunoglobulin (IVIG) $(89 \%)$, and corticosteroid $(73.3 \%)$. A total of 12 patients received anakinra In the severe group, all patients had higher values of KD, PVAS, NLR, cHIS, and CAR than the patients in moderate group. For severe MISC, the area under receiver operating characteristic curve (AUC) was $0.864(95 \%$ confidence interval $[\mathrm{Cl}], 0.729-1)$ for the PVAS, $0.911(95 \% \mathrm{Cl}, 0.827-0.995)$ for the NLR, and $0.853(95 \% \mathrm{Cl}, 0.744-0.963)$ for the CAR, with optimal cut-off values of 3.5 9.05 , and 4.86 , respectively. Thirty-eight $(84.4 \%)$ of the 45 patients met two or more cHIS criteria at the time of their hospitalization; $39 \%$ of these patients were identified as severe group (OR $1.62,95 \% \mathrm{Cl} 1.27-2.13, \mathrm{p}=0.04$ ). At the time of diagnosis, 29 patients with a Kobayashi score greater than 4 were detected, of which 15 required intensive care (OR 2.07, 95\% $\mathrm{Cl} 1.42-3.0, \mathrm{p}=0.00)$.

Conclusion: This study demonstrated that both inflammatory scores (CAR and NLR) and disease activity scores (KS, PVAS and cHIS) can be used to aid the assessment for severity of MISC

REFERENCES:

[1] Webb BJ, et al Lancet Rheumatol. 2020 Dec;2(12):e754-e763.

[2] Feldstein LR et al N Engl J Med. 2020;383(4):334-346

Disclosure of Interests: None declared.

DOI: 10.1136/annrheumdis-2021-eular.2916

\section{POS0083 \\ USEFUL EXPERIENCE OF RHEUMATOLOGISTS FOR UNDERSTANDING OF THE DISEASE ORIGIN AND APPROACHES TO THERAPY IN FIBRODYSPLASIA OSSIFICANS PROGRESSIVA}

I. Nikishina $^{1}$, S. Arsenyeva ${ }^{1}$, V. Matkava ${ }^{1}$, A. Arefieva $^{1}$, M. Kaleda ${ }^{1}$,

T. Pachkoria', Z. Kolkhidova ${ }^{1}{ }^{1}$ V.A. Nasonova Research Institute of Rheumatology, Paediatric, Moscow, Russian Federation

Background: Many monogenic genetic conditions, such as auto-inflammatory diseases (AIDs), have similar clinical manifestations and immunopathogenesis to "classic" rheumatic diseases (RD). Such cases may include Fibrodysplasia ossificans progressiva (FOP), an extremely rare genetic disease, which, according to our previous study and data from other authors 1 , may represent an example of AID with catastrophic heterotopic ossification due to a mutation in the ACVR1 gene. it seems that the experience of rheumatologists, especially children's ones, will be useful in the treatment of FOP.

Objectives: To analyzed the dynamics of clinical manifestations and to therapy approaches including target anti-inflammatory drug Tofacitinib (TOFA) in the one of the world's largest groups of patients (pts) with FOP.

Methods: The study was based on the analysis retrospective and prospective observation of the 35 pts ( 17 males and 18 females) with a verified diagnosis of FOP for the period from 1998 to 2020 . In 9 pts with severe course of FOP TOFA administration were evaluated.

Results: In all 35 pts the diagnosis was verified by "classic" FOP phenotype: malformed great toes in 33 pts $(94,3 \%)$; short malformed thumbs- 8 (22.8\%); peripheral osteochondromas-20 (57.1\%); abnormalities of the cervical spine-32 (91.4\%), multiple heterotopic ossifications-32 (91,4\%). Genetic tests were done in 26 , it confirmed mutation in the ACVR1 gene in $100 \%$. Long term follow-up detected a lot of spondyloarthritis-like signs similar to the manifestation of RD: ankylosis of the facet joints and vertebral bodies (by the type of syndesmophytes) in most pts, sacroiliitis, confirmed by radiological methods (X-ray, CT, MRI), gradual ankylosis in the peripheral joints in 18 (56.4\%), synovitis in large joints in 8 (25\%) pts (knee and hip mostly). In 9 pts with the most difficult course with rapid progression of ossification due to continuous flares despite the NSAIDs and steroids intake, we tried to use TOFA after the approval of the local Ethic Committee. We use the similar dose to randomized trial for JIA (up to $5 \mathrm{mg}$ twice a day). The first patient was 16 y.o. at the time of TOFA administration in December 2019, the age of the other pts was from 2 to 12 y.o. By present time duration of TOFA therapy is from 6 to $15 \mathrm{mo}$. For the previous 6 months before TOFA initiation the number of flares was in average 8 per patient. After 6 months of TOFA treatment the number of new flares decreased to $0-1$, except youngest patient of 2 y.o. in whom the number of flares decreased from 10 to 4 per the same period. In all 9 pts we minimize the dose or completely stop the steroids. New nodes formation stopped immediately in most pts and also the significant motion improvement of large (shoulder) joints were established. Drug tolerance was good in all pts, no AE were registered. But despite the good clinical effect without new heterotopic ossification in our first patient, we found continuous intraskeletal ossification between vertebral bodies, facet and sacroiliac joints in MRI Conclusion: We are confident that the processes of heterotopic ossification in FOP are very similar to new born formation phenomenon in spondyloarthritis and reliable suppression of inflammation can interrupt the progression of the disease. We used similar justifications to our colleagues for the use of anti-cytokine drugs, but used a JAK-kinase inhibitor, it was extremely important the oral rout of drug administration and possibility to escape any injections in FOP. TOFA demonstrated positive effect 
and safety in children with severe course of FOP. It showed their advantages over the use of steroids and possibility to inhibit the rate of progression.

\section{REFERENCES:}

[1] R.Haviv et al. Is fibrodysplasia ossificans progressiva aninterleukin-1 driven auto-inflammatory syndrome? Pediatric Rheumatology (2019) 17:84//doi org/10.1186/s12969-019-0386-6

Disclosure of Interests: None declared.

DOI: 10.1136/annrheumdis-2021-eular.4307

\section{POS0084 \\ SEPTIC ARTHRITIS IN CHILDREN. A LONGITUDINAL POPULATION-BASED STUDY IN WESTERN AUSTRALIA}

J. Nossent ${ }^{1,2}$, D. Preen ${ }^{3}$, H. Keen ${ }^{1,4}$, W. Raymond ${ }^{1}$, C. Inderjeeth ${ }^{1,2}{ }^{1}{ }^{T}$ The University of Western Australia, Medical School, Rheumatology Section, Perth, Australia; ${ }^{2}$ Sir Charles Gairdner Hospital, Rheumatology, Nedlands, Australia; ${ }^{3}$ The University of Western Australia, School of Population and Global Health, Bentley, Australia; ${ }^{4}$ Fiona Stanley Hospital, Rheumatology, Murdoch, Australia

Background: The incidence of Septic arthritis (SA) in adults is rising, but few data are available for children (1). SA symptomatology in young children is often atypical and delayed diagnosis can cause significant morbidity.

Objectives: To describe the incidence, risk factors and long-term outcomes in children hospitalised with septic arthritis (SA) in Western Australia (WA).

Methods: We extracted population-based longitudinally linked administrative health data for patients under 16 years with a first inpatient primary or secondary code of 711.xx (ICD9-CM) and M00.xx (ICD10-AM) in WA for the study period 1990-2010 (to allow a minimum 5 year followup). We report annual incidence rates per 100.000 (AIR), prior conditions during lookback (median 15 months, IQR 5-45) as well as joint and other comorbidities including Charlson comorbidity index $(\mathrm{CCl})$ and standardised mortality rates (SMR) during a median follow-up of 10 years. Age and gender speficic population and mortality rate data were obtained from the Australian Bureau of Statistics.

Results: A total of 891 patients (62\% male, median age 6.4 (IQR 1.9-10.6) years with $34 \%<3$ years of age) had a first admission for SA. AIR was $9.85(\mathrm{Cl} 4.79$ $14.41)$ overall with higher rates in males $(11.9$ vs $7, p<0.01)$ and no apparent period (Figure 1) or seasonal variation. Knees (43.9\%), hips (34.6\%), and ankles $(13.3 \%)$ were most frequently affected with Staphylococci $(49 \%)$ the predominant organism in patients with positive cultures (41.5\%). Prior infections $(40.4 \%)$ and respiratory disease $(7 \%)$ were the main preexisting morbidities. Mean hospital stay was $5.78( \pm 6.4)$ days with ICU admission required in $1.9 \%$, while 30 -day readmittance rate was $10.4 \%$. During follow-up 25 patients $(3 \%)$ had recurrent/ persistent osteomyelitis, nine patients were diagnosed with osteoarthrosis $(1.1 \%)$ and five patients $(0.6 \%)$ underwent joint replacement. More female patients developed new comorbidity $(\mathrm{CCl}>0,34.6$ vs $27.2 \%, \mathrm{p}=0.02)$ including diabetes $(4.2 \%$ vs $0 \%, p=0.001)$, cardiovascular events $(4.2$ vs $1.4 \%, p=0.002)$ and chronic arthritis ( $1 \%$ vs $0, p=0.05)$. While the crude mortality rate was low $(0.3 \%)$ SMR was significantly increased for female patients (10.52, Cl 1.59-41.6).

Conclusion: The statewide incidence of septic arthritis in children in WA is similar to a recent report (1) and did not change over a 20 -year period. In this large population based study, subsequent bone/joint disease occured in $4.6 \%$, while a third of patients developed other comorbidity before the age of 18 . Such (subclinical) comorbidity may thus be a contributing factor to SA development and to the increased mortality risk in female SA patients.

REFERENCES:

[1] Cohen E, Katz T, Rahamim E, Bulkowstein S, Weisel Y, Leibovitz R, Fruchtman Y, Leibovitz E. Septic arthritis in children: Updated epidemiologic, microbiologic, clinical and therapeutic correlations. Pediatr Neonatol. 2020 Jun;61(3):325-330. doi: 10.1016/j.pedneo.2020.02.006

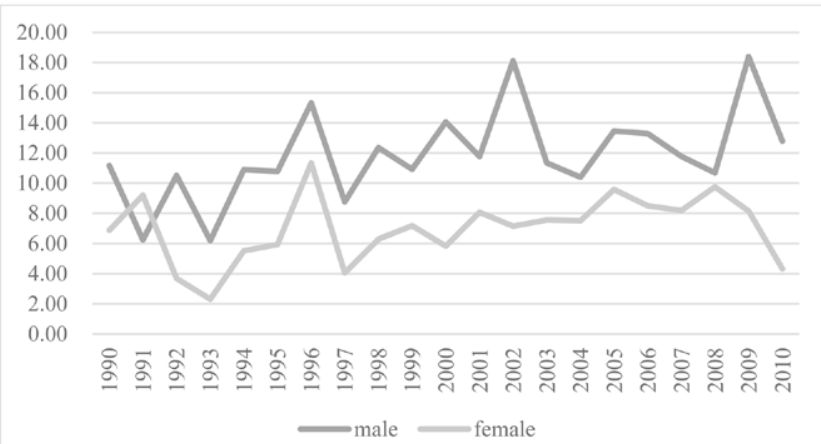

Figure 1. Annual incidence of septic arthritis per 100,000 population $<16$ years in Western Australia over period $1990-2010$ by gender.
Acknowledgements: The authors wish to thank the Arthritis Foundation of WA for their support and would like to acknowledge the support of the Western Australian Data Linkage Branch, the Western Australian Department of Health, and the data custodians of, the Hospital and Morbidity Data Collection, the Emergency Department Data Collection the WA Cancer Register and the WA Death Register for their assistance with data collection.

Disclosure of Interests: None declared.

DOI: 10.1136/annrheumdis-2021-eular.2269

\section{POS0085 \\ EVALUATION OF DURATION OF CLINICAL REMISSION IN CHILDREN WITH NON-SYSTEMIC JUVENILE IDIOPATHIC ARTHRITIS AFTER WITHDRAWAL OF ANTI - TUMOR NECROSIS FACTOR - ALPHA THERAPY}

I. Tsulukiya ${ }^{1}$, E. Alexeeva ${ }^{1,2}$, T. Dvoryakovskaya ${ }^{1,2}$, K. Isaeva $^{1}$, R. Denisova ${ }^{1}$, O. Lomakina ${ }^{1}$, A. Mamutova ${ }^{1}$, A. Chomakhidze ${ }^{1}$, A. Fetisova ${ }^{1}$, M. Gautier ${ }^{1}$, I. Kriulin ${ }^{1,2}$, E. Krekhova ${ }^{1,2}$. 'National Medical Research Center of Children's Health, Rheumatology, Moscow, Russian Federation; ${ }^{2}$ Sechenov First Moscow State Medical University, Pediatric, Moscow, Russian Federation

Background: Juvenile idiopathic arthritis (JIA) is the most common and prevalent rheumatic disease in childhood which is based on a chronic autoimmune inflammation. Inactive disease and remission are now the primary treatment goal in JIA and biologics have been playing an important role to reach this objective. The biologics of the first choice for the treatment of non-systemic JIA are the Tumor Necrosis Factor - alpha (TNF $\alpha$ ) inhibitors; on this therapy patients can achieve clinically inactive disease and long-term remission.

Currently, little is known about when or how to stop TNF $\alpha$ inhibitors, when a good clinical response is achieved, and therefore no guidelines are available.

Objectives: To estimate the length of clinical remission after discontinuation of treatment with TNF $\alpha$ inhibitors in patients with non-systemic juvenile idiopathic arthritis.

Methods: A total of 393 patients with JIA who were treated with TNF $\alpha$ inhibitors at the Rheumatology Department of the National Medical Research Center of Children's Health (Moscow, Russia) were screened for inclusion in this retrospective study.

Patients were treated with etanercept 1 times a week, $0.8 \mathrm{mg}$ per $\mathrm{kg}$ of body weight per dose, with adalimumab $24 \mathrm{mg} / \mathrm{m} 2$ body surface area administered every other week until the end of therapy.Treatment was terminated abruptly. Inactive disease was defined according to the preliminary criteria of Wallace et al.[1]

Results: 77 patients (27-male, 50-female) with a mean age at diagnosis of 4 years (range 1-18 years) were included in the analysis. Of those, 69 of them discontinued TNF $\alpha$ inhibitors due to a long-term remission on treatment, 8 patients as a result of side effects, and there were excluded from our study:

allergic reaction $(n=5)$, development of uveitis $(n=1)$, alopecia $(n=1)$, recurren infection $(n=11)$. The clinical subtypes of JIA were RF-negative polyarticular JIA $-28(40,58 \%)$ oligoarthritis-38 (55,07\%), enthesitis-related arthritis-3 (4,35\%) TNF $\alpha$ inhibitors were started after a mean 46,43 (range 1-144) months of disease. The mean duration of therapy with TNF $\alpha$ inhibitors were 46,63 (range $10-113$ ) months, with a mean duration of remission on medication 40,63 (range 6-107) months before withdrawal of TNF $\alpha$ inhibitors.

$40 / 69(57,97 \%)$ patients did not develop a disease exacerbation and remained in long-term remission off medication-more than 24 months.

Early flares, that is less than 6 months after termination of TNF $\alpha$ inhibitors, were observed in $4 / 69(5,8 \%)$ patients.

$29(42,03 \%)$ patients restarted TNF $\alpha$ inhibitors after exacerbation, due to lack of improvement after no biological DMARDs. All patients in whom TNF $\alpha$ inhibitors were reinitiated responded satisfactorily.

Conclusion: Among patients with JIA in whom TNF $\alpha$ inhibitors were discontinued after inactive disease was achieved, 57,97 \% had disease in clinical remis sion more than 24 months after stopping anti-TNF $\alpha$ therapy. No association was observed between the duration of inactive disease prior to TNF $\alpha$ inhibitors cessation and the time to disease relapse. In addition, we also ob- served no correlation between the risk of flare and the length of anti-TNF $\alpha$ therapy after inactive disease was achieved. In our population, TNF $\alpha$ antagonists were withdrawn a median of 38 (4-107) months after inactive disease was achieved. Data from our experience with anti-TNF $\alpha$ agents in the treatment of JIA suggest that $57,97 \%$ of patients can be successfully withdrawn from TNF $\alpha$ antagonists for at least 24 months.

\section{REFERENCES:}

[1] Wallace CA, Giannini EH, Huang B, Itert L, Ruperto N, for the Childhood Arthritis and Rheumatology Research Alliance (CARRA), the Pediatric Rheumatology Collaborative Study Group (PRCSG), and the Paediatric Rheumatology Interna- tional Trials Organisation (PRINTO). American College of Rheumatology provisional criteria for defining clinical in- active disease in select categories of juvenile idiopathic arthritis. Arthritis Care Res (Hoboken) 2011;63:929-36.

Disclosure of Interests: None declared.

DOI: 10.1136/annrheumdis-2021-eular.2257 